Meta

Journal des traducteurs

Translators' Journal

\title{
L’initiation à la traduction économique
}

\section{Jean Delisle}

Volume 33, numéro 2, juin 1988

L'enseignement de la traduction au Canada - Teaching Translation in Canada

URI : https://id.erudit.org/iderudit/002515ar

DOI : https://doi.org/10.7202/002515ar

Aller au sommaire du numéro

Éditeur(s)

Les Presses de l'Université de Montréal

ISSN

0026-0452 (imprimé)

1492-1421 (numérique)

Découvrir la revue

Citer cet article

Delisle, J. (1988). L'initiation à la traduction économique. Meta, 33(2), 204-215.

https://doi.org/10.7202/002515ar d'utilisation que vous pouvez consulter en ligne.

https://apropos.erudit.org/fr/usagers/politique-dutilisation/ 


\title{
L'INITIATION À LA TRADUCTION ÉCONOMIQUE
}

JEAN DELISLE

Université d'Ottawa, Ottawa, Ontario

\begin{abstract}
À la fin d'une communication présentée à l'Université du Québec à Montréal en 1980, j'avais formulé le vœu suivant :

Que s'intensifie la réflexion sur la méthodologie des cours pratiques de traduction et que ce thème fasse l'objet d'un mini colloque qui réunirait les pédagogues désireux de mettre en commun leur expérience de l'enseignement ${ }^{1}$.
\end{abstract}

Six ans plus tard, je constate que mon souhait est resté lettre morte. Que j'envie les terminologues! Les fiches de mon précis d'histoire de la traduction au Canada ${ }^{2}$ me révèlent que, depuis 1970, les terminologues canadiens ont tenu le nombre impressionnant de vingt-deux colloques et journées d'études, une moyenne de 1,46 colloque par année. Ripailles de mots et de redites, diront les méchantes langues, ennemies de ce genre de rencontres. Occasions propices à une réflexion sur tous les aspects de la terminologie, rétorqueront les infatigables organisateurs de ces forums. Force est de reconnaître qu'au cours des quinze dernières années, la terminologie s'est hissée au rang de discipline autonome en sachant se doter d'un corps de doctrine cohérent et de principes terminographiques éprouvés.

Jamais, à ma connaissance, les professeurs chargés des cours pratiques de traduction n'ont tenu un seul colloque afin de discuter spécifiquement de questions méthodologiques, de partager leur expérience concrète de l'enseignement et de mettre en commun leurs réussites et leurs échecs dans ce domaine. Sait-on seulement ce qui se fait concrètement dans la salle de classe ? Sait-on comment s'enseigne la traduction d'une école à l'autre ? Je serais bien embêté de dire comment mes collègues des autres universités enseignent la traduction économique, et eux ne savent probablement pas non plus comment je procède. Ne peut-on pas pousser la réflexion sur l'enseignement de la traduction au-delà de la structuration des programmes ? Est-il déraisonnable de souhaiter que les pédagogues s'interrogent sur les modalités de l'apprentissage de la traduction dans et en dehors de la salle de classe ? Les méthodes d'enseignement ont-elles atteint un tel degré d'efficacité qu'elles ne sont plus perfectibles? Les faiblesses relevées par les employeurs chez les diplômés de nos écoles sont-elles attribuables uniquement aux lacunes de l'enseignement préuniversitaire? Ne peut-on pas s'interroger en toute lucidité et en toute honnêteté sur la valeur de nos méthodes d'enseignement? Se pourrait-il qu'elles aussi présentent des faiblesses et soient améliorables?

Autant de question qui pourraient alimenter des discussions passionnées (et passionnantes) lors d'un colloque axé sur la didactique de la traduction professionnelle. En attendant la tenue d'un tel forum ${ }^{3}$, j'applaudis à l'initiative de mon collègue, Robert Larose, qui, en assurant la coordination de ce numéro spécial offre l'occasion aux pédagogues de la traduction au Canada de réfléchir sur l'efficacité de leurs méthodes d'enseignement, de proposer des techniques nouvelles d'apprentissage et de partager leur expérience d'enseignants. Pour ma part, j'ai choisi de traiter d'un sujet qui me tient tout 
particulièrement à cour : les aspects méthodologiques des séminaires pratiques de traduction.

Dans la suite de cet article, je décrirai la façon dont j'ai structuré mon séminaire d'initiation à la traduction économique. Ce séminaire de premier cycle (baccalauréat), je le donne depuis sept ans à l'École de traducteurs et d'interprètes de l'Université d'Ottawa. J'ai voulu donner à mon article la forme d'un témoignage tiré de mon expérience personnelle. D'aucuns trouveront peut-être mes propos " terre à terre " et près de la réalité quotidienne des enseignants ; c'est que je ne m'adresse pas aux théoriciens de la traduction, mais d'abord et avant tout aux " pédagotrads" comme dirait Jean-Paul Vinay. Mon intention est, en fait, de montrer comment il est possible d'appliquer aux séminaires de traduction spécialisée la méthode par objectifs d'apprentissage que j'ai déjà préconisée pour l'enseignement de la traduction générale ${ }^{4}$.

La définition d'objectifs est indissociable de toute véritable stratégie pédagogique et rejoint la préoccupation des didactiques des langues, avec lesquels, soit dit en passant, les pédagogues de la traduction professionnelle ont beaucoup plus d'affinités qu'avec les linguistes. Qu'il suffise de rappeler à cet égard la définition de la notion "d'objectif " proposée par Robert Galisson et Daniel Coste dans leur excellent Dictionnaire de didactique des langues:

En didactique des langues, comme dans d'autres domaines, la notion d'" objectif ", dont on ne s'était souvent que trop peu soucié, reprend une fonction cardinale (il en va de même pour celle de "public"). Les problèmes touchant au choix des contenus d'enseignement, aux progressions et à l'évolution tendent à être repensés en rapport avec les objectifs assignés à l'apprentissage. D'autre part — mais de façon nécessairement complémentaire - on s'attend que les objectifs soient définis de façon opératoire, en termes de tâches à accomplir, de capacités à montrer, de comportement à maîtriser 5 .

À tout objectif est associé un ou plusieurs " procédés ", c'est-à-dire des " manières de faire qui règlent les rapports maître-élèves dans des situations scolaires précises, en vue de la présentation, de l'assimilation ou du contrôle de la matière à enseigner. La reconstitution de texte, l'exercice structural, la dictée préparée, le commentaire d'image, le dialogue, etc., sont des procédés ${ }^{6}$ " courants en enseignement des langues. Nous verrons dans la suite du texte quels procédés il est possible de concevoir et d'appliquer en pédagogie de la traduction.

Mon séminaire d'initiation à la traduction économique s'organise autour des cinq objectifs d'apprentissage suivants :

1. Assimiler les notions fondamentales de l'économie.

2. Assimiler la terminologie de base de l'économie.

3. Assimiler la phraséologie propre aux textes économiques.

4. Apprendre à se documenter sur le sujet.

5. Développer l'aptitude à traduire des textes économiques.

Pour atteindre ces cinq objectifs généraux, j'ai recours à une vingtaine de "procédés " ou " techniques d'apprentissage ». On touche ici à l'aspect "créateur » de l'enseignement. Enseigner, tout comme traduire, est un art qui exige à la fois des connaissances, du talent et, avouons-le, le feu sacré. Personnellement, j'éprouve autant de satisfaction à préparer un cours qu'à le donner, car la préparation de cours me fournit l'occasion d'innover et d'exercer une certaine forme de créativité en recherchant la façon la plus efficace pour atteindre les objectifs définis pour tel cours ou tel séminaire.

J'ouvre ici une parenthèse afin d'énumérer les dix qualités du "bon professeur" selon les étudiants de l'Université de Montréal. Il est permis de croire que le sondage, dont le résultat figure ci-après, aurait abouti aux mêmes conclusions s'il avait été mené 
dans tout autre établissement d'enseignement supérieur. Par ordre d'importance, les qualités reconnues aux bons professeurs d'université sont les suivantes :

1. Connaissance de sa matière (connaissances renouvelées et à jour).

2. Méthodique et structuré (prépare, organise et présente la matière des cours de façon méthodique et structurée; esprit de synthèse).

3. Fait le lien entre la théorie et la pratique.

4. Clarté de l'exposé.

5. Favorise le développement intellectuel de l'étudiant (incite à la recherche, développe le sens critique et la curiosité intellectuelle).

6. Goût d'enseigner (intéressé à ce qu'il enseigne, enthousiaste).

7. Présentation intéressante de la matière.

8. Respect des étudiants (sait échanger avec les étudiants; est ouvert à leurs suggestions, critiques et opinions).

9. Évaluation juste (évalue les étudiants de façon objective et impartiale ; précise le mode d'évaluation).

10. Disponibilité (accessible en dehors des heures de cours, prêt à aider les étudiants et à leur fournir les ressources nécessaires) ${ }^{7}$.

On remarque que la présentation "méthodique et structurée » de la matière enseignée (point $\mathrm{n}^{\circ} 2$ ) est considérée par les étudiants comme l'aspect le plus important de l'enseignement après la maîtrise du sujet par le professeur. C'est donc dire l'importance accordée à la structuration des cours. On note également que les étudiants jugent plus importante la "présentation intéressante de la matière" (point $\mathrm{n}^{\circ} 7$ ) que l'équité de l'évaluation (point $n^{\circ} 9$ ). Par "présentation de la matière ", il faut entendre, bien sûr, l'attitude du professeur en classe (qualité de l'élocution, débit, aptitude à susciter la participation des étudiants, etc.), mais aussi l'originalité et l'efficacité des moyens mis en ouvre pour faire assimiler la matière au programme (qualité, diversité et pertinence des exercices pratiques, utilisation d'aides audiovisuelles, etc.).

Je referme cette parenthèse pour revenir aux procédés associés à chacun des objectifs du séminaire d'initiation à la traduction économique. Il y a lieu de préciser que, même si tous ces objectifs font l'objet d'exercices spécifiques, ils sont menés plus ou moins de front du début à la fin du séminaire. En outre, certains procédés sont utilisés en classe, d'autres prennent la forme de travaux individuels à faire à la bibliothèque ou chez soi. (Cf. le tableau récapitulatif à la fin de cet article.)

\section{ASSIMILER LES NOTIONS FONDAMENTALES DE L'ÉCONOMIE}

Trois procédés :

a) Exposés magistraux.

b) Lecture d'un ouvrage de vulgarisation sur l'économie.

c) Utilisation d'aides audiovisuelles.

Les exposés magistraux ont lieu généralement au début des premières séances en classe. Chacune de ces séances dure une heure et demie. Ces exposés servent à préciser certaines notions soit en les définissant, soit en donnant des explications pertinentes. Exemples de notions ainsi traitées : most favoured clause (clause de la nation la plus favorisée) ; financial market (marché financier) vs money market (marché monétaire); Law of diminishing returns (Loi des rendements décroissants) ; nominal GNP (PNB nominal) vs real GNP (PNB réel); clearing house (chambre de compensation).

L'assimilation des notions se poursuit hors de la salle de classe par la lecture d'un ouvrage de vulgarisation. Il n'est pas facile de trouver un ouvrage qui aborde à la fois la macro-économie, la micro-économie, le rôle des banques et de l'État tout en présentant les grands organismes internationaux. J'aurais pu imposer la lecture de plusieurs ma- 
nuels complémentaires d'initiation à l'économie, mais, compte tenu de la durée du séminaire (45 heures), j'ai préféré m'en tenir à un seul ouvrage. J'ai choisi celui de Léon Courville, l'Économie démystifiée (Montréal, Chenelière/Stanké, $2^{\mathrm{e}}$ édition, 1983). En trois cents pages et soixante et onze courts chapitres, les auteurs (car il s'agit en fait d'un collectif) décrivent les rouages de l'économie moderne à partir de faits d'actualité familiers au lecteur. Exemples de têtes de chapitres : Faut-il nationaliser la Société des alcools? Un toit pour le stade olympique? Les joueurs de base-ball sont-ils trop payés ? Le vin dans les restaurants : doit-on crier au voleur?

L'ouvrage réunit des articles publiés par un groupe de professeurs d'économie et de finance de l'École des hautes études commerciales (HEC) de Montréal dans les deux journaux de la métropole : le Devoir et Dimanche-Matin. Malgré quelques faiblesses de rédaction - j'y reviendrai - ce recueil d'articles remaniés m'a semblé répondre aux besoins des étudiants d'un séminaire d'initiation à la traduction économique. Le journalisme économique est un des trois niveaux de fonctionnement des textes d'économie, les deux autres étant l'exposé théorique (assorti de nombreuses formules algébriques et d'opérations mathématiques rebutantes à souhait pour tout néophyte) et le manuel d'enseignement. Chacun de ces niveaux se caractérise, indépendamment du vocabulaire, par certaines variantes stylistiques ${ }^{8}$. En outre, les préoccupations didactiques des auteurs des manuels d'initiation à l'économie et des journalistes de la presse économique se concilient bien avec celles du professeur de traduction économique qui cherche à étendre les connaissances des apprentis traducteurs dans ce domaine.

En ce qui concerne la qualité de la langue de l'Économie démystifiée, je la considère acceptable. On y retrouve la phraséologie courante dans les textes de cette nature et dont les étudiants sont invités à s'imprégner. Exemples : " le rendement de la production s'effrite"; " être insensible à une variation de prix "; " la dépréciation de notre monnaie entame la confiance des investisseurs"; " la surchauffe de l'économie "; "gonfler la valeur des titres".

Par contre, l'ouvrage comporte un petit nombre d'erreurs de forme malheureusement très répandues dans la presse écrite au Canada français. Ces erreurs, le présidentfondateur de l'Académie canadienne-française, $M$. Victor Barbeau, les a farouchement combattues tout au long de sa carrière de professeur de "français langue étrangère " aux HEC ${ }^{9} . .$. Exemples : " magasin à rayons » (grand magasin), "en 1981, alors que » (anglicisme de syntaxe indéracinable au Canada français) ; "expertise " (compétence, savoirfaire, spécialité) ; "comptes à recevoir" (comptes-clients); "comptes à payer " (comptes-fournisseurs) ; inventaire " (au sens de stock) ; " couper » les taxes (au lieu de réduire ; pourtant, on dit bien "sabrer " dans les dépenses...) ; " domestique " (national, intérieur, du pays, canadien) ; "éligible» (employé abusivement pour admissible). L'effet corrosif de l'anglais sur le français se manifeste donc aussi sous la plume des économistes qui ont participé à ce collectif. Leur vocabulaire spécialisé, toutefois, est juste et précis. J'aurais préféré bien sûr utiliser un ouvrage qui, sans comporter ces scories, eût réuni les mêmes qualités que l'Économie démystifiée. Les qualités que je reconnais à cet cuvrage sont les suivantes : simplicité, concret, prix abordable $(6,95 \$)$, bon niveau de vulgarisation, abondance de la matière, date récente de publication, traite des institutions québécoises et canadiennes tout en présentant les grands organismes européens et internationaux.

Comme il est indispensable en traduction d'accorder une importance égale à la forme et au fond, je pense avoir trouvé une façon de mettre à profit ces erreurs de forme. Les ayant relevées lors d'une lecture à la loupe de l'ouvrage, j'en fais la matière des toutes premières séances du séminaire ; mes années d'enseignement de la traduction m'ont appris que ce sont des erreurs dont les étudiants émaillent allègrement leurs copies. 
Cette opération d'épuration se transforme donc en exercice de prévention. En pédagogie, tout est récupérable!

Enfin, l'assimilation des notions d'économie peut se faire au moyen d'aides audiovisuelles. Je songe, par exemple, à des films ou à des diaporamas sur le fonctionnement de la bourse, le jeu de la loi de l'offre et de la demande, les taux de change, ou sur tout autre sujet relatif à l'économie. La télévision présente assez souvent des émissions fort bien faites sur des questions économiques. Grâce aux nouvelles vidéocassettes qui envahissent nos foyers depuis quelque temps, il est facile d'enregistrer ces émissions et de les mettre ensuite à la disposition des étudiants. La visualisation peut se faire en dehors des cours dans les salles d'audiovisuel dont sont dotées toutes les universités. On peut aussi concevoir que ces enregistrements magnétoscopiques circulent entre les professeurs de traduction économique d'universités différentes. (Il est permis de rêver !) À la longue, il se formerait une banque d'émissions dans laquelle chaque professeur pourrait puiser. Est-ce rêver en couleur de penser que le pédagogue de la traduction peut, lui aussi, tirer profit des progrès de la technologie moderne?

\section{ASSIMILER LA TERMINOLOGIE DE L'ÉCONOMIE}

Quatre procédés :

a) Remise d'un vocabulaire photocopié de l'économie.

b) Exposés magistraux sur des difficultés de nature terminologique.

c) Renvoi à des études terminologiques publiées.

d) Recherches terminologiques ponctuelles.

L'acquisition de la terminologie de l'économie se fait évidemment en partie par la lecture de l'Économie démystifiée. J'ai relevé dans cet ouvrage pas moins de trois cents termes en contexte, certains étant même accompagnés d'une définition (comme c'est souvent le cas dans les écrits de vulgarisation ou journalistiques). On y retrouve une bonne part du vocabulaire de base de l'économie, depuis l'" appréciation de la monnaie " jusqu'à la notion de "cartel ", en passant par celles de "politique fiscale", "ménage ", "liquidités" et "taux d'escompte".

Chaque étudiant reçoit en outre un vocabulaire de cent vingt-cinq termes environ accompagnés d'une définition formelle. (Certains de ces termes font l'objet des explications lors des exposés magistraux sur les notions d'économie.) Exemples de termes figurant dans ce recueil de mots clés de l'économie : special drawing rights (droits de tirage spéciaux), snake (serpent), reserves (réserves), cycle (cycle), central bank (banque centrale).

Dans des exposés magistraux, je traite, par exemple, des aspects suivants du vocabulaire économique : terme ayant une extension de sens différent en français et en anglais (operating budget se traduit par " budget d'exploitation " s'il s'agit d'une entreprise privée, mais par " budget de fonctionnement » dans le cas d'un organisme public), faux amis (depreciation = dépréciation vs amortissement), synonymie (share (GB), stock (US), néologie (stagflation, taux désaisonnalisé (CDN), sigles et abréviations (PIB, FECOM, ECU, FOB, FMI), etc.

Une liste d'études terminologiques publiées dans des revues ou bulletins tels que l'Actualité terminologique, META, la Banque des mots, Vie et langage, Informations terminologiques est aussi remise aux étudiants. Il leur est demandé de les lire durant le semestre et d'en assimiler le contenu qui est évidemment matière à examen. Dans leur Bibliographie sélective du traducteur - Commerce et économie (Montréal, Linguatech, 1977 ; Addendum, 1979, 30 p.), Paul Horguelin et Jean-Claude Gémar citent près de soixante-quinze études de ce genre dans des domaines tels que la banque, la bourse, la comptabilité, l'emploi, la gestion, l'organisation des entreprises, la publicité. Chaque année, je choisis celles qui sont les plus pertinentes aux textes que je donne à traduire. 
La recherche terminologique ponctuelle peut être un autre moyen auquel le professeur peut recourir pour atteindre le deuxième objectif de l'initiation à la traduction économique. Ces brèves études peuvent porter, par exemple, sur les acceptions du terme staple ou sur la distinction à faire entre "main-d'œuvre " et "population active ", ces deux mots pouvant traduire, selon le contexte, labor force. Faute de temps, je laisse ce genre de travaux pratiques au professeur de terminologie.

\section{ASSIMILER LA PHRASÉOLOGIE PROPRE AUX TEXTES ÉCONOMIQUES}

Deux procédés :

a) Exercices de phraséologie.

b) Lectures parallèles (textes originaux et traductions).

Par phraséologie, j'entends l'ensemble des expressions et particularités syntactiques propres à l'usage admis dans la langue économique. Cela inclut aussi bien les emplois métaphoriques de certains mots de la langue courante que tout le vaste champ de la cooccurrence et de la collocation ${ }^{10}$. Exemples : l'effritement du pouvoir d'achat, le resserrement du crédit, la spirale inflationniste, le rétrécissement des marchés d'exportation, la flambée des prix, donner une forte impulsion aux secteurs mous de l'économie, etc.

Les exercices de phraséologie, qui sont en fait des exercices de maniement de la langue économique, se font collectivement en classe. Chaque exercice renferme une dizaine de passages (ce que j'ai appelé ailleurs des "unités textuelles"1") extraits d'un texte économique et qu'il s'agit de traduire. La langue de l'économie se prête facilement au découpage en unités textuelles et en cela, elle ne diffère pas tellement des autres langues de spécialité. Exemples traduits et commentés en classe :

The textile industry has not been sharing the boom condition.

L'industrie textile n'a pas eu sa part de la prospérité générale.

Moins longs au début, les passages se rapprochent du paragraphe au fur et à mesure que le séminaire progresse.

Economic growth in Canada and almost all industrial countries slowed down in 1982. The growth in personal spending decelerated while inventory accumulation, which had provided a significant boost to output growth in the first half, did not increase. No sector came forward to inject new stimulus into the pace of economic activity, thus accentuating the weakness of the present recovery.

La croissance économique a fléchi en 1982 au Canada comme dans presque tous les pays industrialisés. L'expansion des dépenses personnelles a ralenti tandis que la formation de stocks qui avait fortement stimulé la croissance de la production au premier semestre stagnait. Aucun secteur ne venant ranimer l'activité économique, la lenteur de la reprise actuelle s'est accentuée.

Ces exercices, occasion de nombreux commentaires de tous ordres, se révèlent utiles pour faire acquérir aux futurs traducteurs les tournures typiques de la langue économique, les façons d'exprimer les phénomènes économiques. Ils sont aussi l'occasion d'un enrichissement du vocabulaire ; on s'attardera, par exemple, aux très nombreuses façons d'exprimer un mouvement de hausse ou de baisse sur le modèle proposé, il y a plus de vingt-cinq ans, dans le fameux Vocabulaire baromètre dans le langage économique.

Les mots étudiés présentent [...] une telle vitalité, font remarquer les auteurs, que d'une langue à l'autre il est rare qu'ils offrent cette coïncidence rigoureuse qu'on rencontre souvent dans le vocabulaire purement technique [et ils] ont aussi une telle fréquence dans tous les 
textes qui touchent à l'économie ou à la finance - études de la conjoncture, études de marché, chroniques boursières, etc., - qu'en résolvant une difficulté on sera sûr d'en avoir résolu par avance maintes autres ${ }^{12}$.

C'est ce vers quoi il faut tendre en pédagogie de la traduction : s'attarder à tous les aspects (lexicaux, phraséologiques, stylistiques, thématiques) les plus récurrents d'un domaine, et ce afin d'accroître l'efficacité de l'enseignement.

La lecture parallèle de textes originaux d'économie et de leurs traductions est un autre moyen de s'imprégner de la phraséologie. Il ne suffit pas, toutefois, de conseiller aux étudiants de faire de telles lectures comparées, car bien peu d'entre eux pousseront le zèle jusqu'à diriger leurs pas vers la bibliothèque... Il est donc essentiel de doubler cet exercice d'un travail de repérage d'une difficulté de réexpression liée à la traduction économique. Exemple : relever un ou plusieurs passages renfermant un verbe de mouvement ou de destination, une métaphore filée (figure dont font grand usage les rédacteurs de textes économiques), des exemples de divergences entre l'anglais et le français en ce qui concerne les abréviations utilisées dans les tableaux, etc. Ainsi, il arrive souvent que, par manque d'espace, l'anglais se contente d'écrire dans les tableaux (000's) au lieu de thousands of dollars. Le français admet " en milliers de dollars ", " milliers de dollars " et " milliers de $\$$ ".

\section{APPRENDRE À SE DOCUMENTER SUR LE SUJET}

Deux procédés :

a) Présentation des principales sources documentaires.

b) Recherches documentaires.

Rares sont les professeurs qui ne donnent pas au début d'un séminaire des indications bibliographiques. Le professeur de traduction commerciale et économique a la chance de pouvoir compter sur une bibliographie sélective et sectorielle s'adressant à la fois aux étudiants en traduction et aux traducteurs professionnels. "À l'aide de cet inventaire et des cotes d'appréciation [indispensable pour guider le choix des apprentis traducteurs], l'utilisateur peut établir rapidement la liste des ouvrages disponibles et choisir ceux qui seront essentiels ou utiles à son travail, en fonction de la nature et de l'ampleur du texte à traduire ${ }^{13}$."

Au début de chaque année, je remets à mes étudiants un exemplaire de trois petits lexiques bilingues d'une trentaine de pages chacun distribués gratuitement par le Bureau des traductions du Secrétariat d'État : Lexique économie, Lexique finance, Lexique comptabilité.

On peut aussi concevoir des exercices offrant aux étudiants l'occasion de consulter quelques ouvrages de référence jugés particulièrement fiables et utiles. Exemple : demander aux étudiants de retrouver la source d'une étude terminologique donnée en suggérant un certain nombre de pistes de recherche ou décrire le contenu de quelques ouvrages de consultation.

\section{DÉVELOPPER L'APTITUDE À TRADUIRE DES TEXTES ÉCONOMIQUES}

Six procédés :

a) Exposés sur des difficultés de nature grammaticale ou stylistique.

b) Exercices en classe sur ces difficultés.

c) Traduction de textes économiques.

d) Discussion en classe des traductions annotées.

e) Remise de corrigés.

f) Journées de traduction. 
Les exposés et exercices sur les difficultés de nature grammaticale ou stylistique rencontrées fréquemment en traduction économique portent, par exemple, sur les verbes de mouvement et les verbes de destination qu'il faut traduire avec prudence en passant de l'anglais au français. L'étude de cette difficulté pourra être l'occasion d'un enrichissement de la "palette expressive " des apprentis traducteurs grâce à l'examen des nombreuses possibilités de la langue française pour exprimer un mouvement ou une destination (en progression sur ; marque un recul de ... par rapport à ... ; a été ramené à ; fut porté de ... à ..., etc.). Chaque "niveau " de texte (théorique, didactique, journalistique) présente des traits particuliers du point de vue stylistique, qu'il est bon de faire ressortir. C'est ici qu'il convient d'aborder les problèmes d'articulation, la nominalisation, les types de phrases (rares occurrences de l'impératif dans les textes économiques théoriques), usage respectif des tournures passives en français et en anglais, etc. Il va de soi que ces exposés sont assortis d'exemples et d'exercices d'application.

En principe, je ne fais jamais traduire de textes suivis en classe. J'estime que c'est un procédé dépassé que de distribuer aux étudiants un texte à traduire au début d'un " cours " et de leur demander d'en traduire une phrase à tour de rôle. Un texte quel qu'il soit ne se traduit pas en phrases détachées. Il serait trop long de débattre de cette question ici. Disons simplement que la traduction collective d'un texte suivi est un exercice qui comporte plus d'inconvénients que d'avantages du triple point de vue des contenus d'enseignement (presque nuls), de la méthodologie (absence de méthode, les difficultés étant abordées au hasard des textes) et des principes pédagogiques (ces séances de traduction sont démotivantes et souvent mortellement ennuyantes). Pour progresser, la pédagogie de la traduction doit à tout prix délaisser cette technique artisanale au profit d'un enseignement universitaire mieux structuré et plus exigeant.

C'est en classe, par contre, que sont commentés les travaux pratiques annotés (et généralement notés). Chaque étudiant s'étant heurté aux difficultés des textes à traduire, ayant essayé par lui-même de triompher des difficultés, les erreurs commises (comme les solutions heureuses) prennent toute leur valeur pédagogique. Dans un séminaire de quarante-cinq heures, je donne à traduire une douzaine de textes d'environ 300 à 350 mots. C'est peu et c'est beaucoup. C'est peu si l'on se place du point de vue des besoins des étudiants; c'est beaucoup si l'on tient compte des heures de corrections qu'exige l'annotation minutieuse d'une quarantaine de copies par semaine. La solution serait de limiter le nombre des admissions. Le choix contraire risque de faire perdre toute valeur et toute crédibilité aux diplômes universitaires de traduction. Il serait dommage que ces diplômes connaissent le même sort que les assignats de la Révolution de 1789 ! Sans être abusivement élitiste, je suis partisan d'une sélection rigoureuse des candidats aux diplômes en traduction. C'est aussi par là que passe la reconnaissance de fait de la profession. Il $\mathrm{y}$ aurait beaucoup à dire à ce sujet et de nombreuses leçons à tirer de l'histoire de la traduction. Mais qui écoute les historiens de la traduction... ?

Le corrigé peut avoir lui aussi une valeur pédagogique bien que les avis soient partagés à ce sujet. Certains professeurs " déconseillent fortement la remise de corrigés " invoquant comme arguments que " les étudiants ne [les] lisent pas", que « la remise d'un corrigé tend à faire croire qu'il n'y a qu'une seule traduction possible et correcte d'un texte (celle du professeur) ", qu' i il est beaucoup plus formateur d'établir le corrigé avec les étudiants, en remettant leurs copies " et, enfin, que "les corrigés servent davantage aux étudiants des années suivantes qu'à ceux auxquels ils sont destinés ${ }^{14}$ ".

D'autres enseignants, au contraire, trouvent les corrigés utiles :

...nous ne voyons pas pourquoi le professeur éviterait de remettre sa propre version de la traduction, ou toute version établie par quelqu'un d'autre, à moins (qu'il veuille) utiliser les mêmes textes jusqu’à la fin de sa carrière [...] Soulignons à ce sujet combien il serait injusti- 
fié, pour écarter la remise aux étudiants d'un corrigé, d'invoquer l'inutilité de ce dernier, ou de lui prêter des dangers. Contrairement à ce que certains préjugés mènent parfois à affirmer, la grande majorité des étudiants lisent attentivement les corrigés, et bien peu d'entre eux sont assez naifs pour croire que la seule traduction possible et correcte est toujours celle du professeur ${ }^{15}$.

Au sujet des corrigés, mon opinion est la suivante et se fonde sur l'observation de la réaction des étudiants en classe.

1. Les étudiants lisent les corrigés si ceux-ci leur sont remis avant leurs copies annotées et si on leur laisse le temps d'en prendre connaissance. Un corrigé remis en vitesse à la fin d'un séminaire a relativement peu de valeur.

2. Le corrigé permet de faire ressortir les qualités d'une bonne traduction, donc les attentes du professeur, si celui-ci prend la peine de le lire et de le commenter à la fin d'une séance de correction.

3. Afin d'atténuer le caractère "dogmatique " de cet instrument pédagogique, il est facile d'y intégrer quelques-unes des bonnes solutions relevées dans les copies d'étudiants et même d'en féliciter les auteurs devant la classe ; toujours valorisants, les compliments augmentent la motivation au travail.

4. En se voyant "publiés ", les étudiants n'ont pas tendance à croire que le professeur essaie d'imposer sa solution comme la seule valable.

5. Le corrigé peut aussi être une traduction dite " officielle » ou " publiée ». Il suffit d'en indiquer la source. Les étudiants sont alors à même de constater que les qualités que leur professeur reconnaît à une bonne traduction sont aussi celles que cherchent à atteindre les traducteurs professionnels. C'est un moyen de rétrécir l'écart entre les exigences universitaires et celles du milieu du travail.

6. Il ne me semble pas indispensable de remettre un corrigé pour tous les textes à traduire. Ce procédé pédagogique trouve sa plus grande utilité lors de l'annotation des premiers textes traduits dans un domaine nouveau.

7. Parce qu'il propose de bonnes solutions, le corrigé fait contrepoids aux erreurs relevées dans les copies d'étudiants. Dans un contexte pédagogique, s'il est indispensable d'informer (l'information est à la base de la formation) les étudiants sur ce qu'il convient de ne pas faire en traduction, il est tout aussi important de ne pas adopter une attitude exclusivement négative et de donner des exemples de la façon dont on peut rendre correctement un texte dans une autre langue.

8. Le corrigé, enfin, se révèle indispensable pour illustrer certaines qualités "supraphrastiques "d'un texte : enchaînement des idées, cohésion d'un paragraphe, concision, tonalité, etc. La correction d'un simple faux ami peut très bien se faire sans référence à tout un texte.

Pour toutes ces raisons, j'estime que le corrigé est un instrument de formation dont la valeur pédagogique est réelle; il serait dommage de s'en priver. Tout dépend de la manière dont on l'utilise dans la salle de cours. Quant à l'argument selon lequel il peut se créer un "marché noir" des corrigés, j'avoue qu'après avoir corrigé quarante versions d'un même texte, je ne suis pas fâché de changer de sujet.

La journée de traduction est une autre technique permettant de développer l'aptitude à traduire. Il s'agit d'organiser une journée complète de traduction comme en vivent les traducteurs professionnels... à quelques différences près. Pour résumer brièvement en quoi consiste une telle journée, disons qu'un même texte de mille à mille cinq cents mots est remis en début de journée à chaque étudiant qui le traduit seul sur sa machine à écrire. La journée est évidemment ponctuée des " pauses syndicales » réglementaires. Ce procédé, qui permet de faire du volume et d'acquérir un peu de vitesse, donne un aperçu des conditions de travail qui attendent les futurs traducteurs. La journée de 
traduction est profitable surtout vers la fin d'un cours ${ }^{16}$ et offre l'occasion d'une révision générale.

Voilà, très sommairement décrits, dix-sept procédés d'apprentissage propres à atteindre les cinq grands objectifs pédagogiques de l'initiation à la traduction économique. Dans le tableau récapitulatif qui accompagne cet article, je les ai répartis en deux colonnes : ceux que j'applique en classe et ceux qui exigent un travail individuel de la part des étudiants en dehors de la salle de cours.

Le fait de structurer l'enseignement autour de ces objectifs et procédés permet de cerner assez systématiquement tous les principaux aspects des textes économiques à traduire. Cette méthode facilite la délimitation de ce que Robert Galisson et Daniel Coste appellent les "contenus d'enseignement", tout en rendant possible l'établissement d'une "progression" dans la matière enseignée.

Du point de vue du "contrôle" des connaissances acquises, cette méthode présente en outre l'avantage, non négligeable, de multiplier les bases d'évaluation des étudiants. Il est ainsi possible de contrôler séparément ce qui relève de l'acquisition pure et simple de connaissances (notions d'économie, vocabulaire, sources documentaires) et ce qui relève du maniement du langage, c'est-à-dire le savoir-faire du traducteur (aptitude à interpréter le sens des textes, à articuler les idées, à manier la phraséologie propre au domaine, à préserver les effets stylistiques, les niveaux de langue, à adapter les textes aux destinataires).

Puisque " traduire est un savoir-faire (interpréter et réexprimer) reposant sur un double savoir (linguistique et encyclopédique) ${ }^{17}$, il importe, lors des tests ou examens, de faire porter l'évaluation à la fois sur les connaissances et sur les aptitudes acquises. C'est pourquoi tout ce qui a été vu durant le séminaire est matière à examen : le vocabulaire, la matière ayant fait l'objet des exposés et des exercices (phraséologiques, grammaticaux, stylistiques, terminologiques), les corrections des traductions annotées et l'ouvrage de vulgarisation. (Dix questions portant sur le contenu du livre sont remises aux étudiants qui doivent les préparer en vue de l'examen.) Pour les examens semestriels, d'une durée de trois heures, les étudiants n'ont droit à aucune documentation. Outre une traduction d'environ 150 à 200 mots, ils ont à répondre à un certain nombre de questions dont voici quelques exemples :

1. Définissez succinctement les dix notions d'économie ci-dessous et donnez leurs équivalents français :
a) economic agent
b) free trade
f) market price
c) money market
g) exchange rate
d) business cycle
e) economic balance sheet
h) economies of scale
i) money supply
j) special drawing rights

2. Distinguez les notions suivantes et donnez l'équivalent français de chacune d'elles :

a) constant dollar gross national product current dollar gross national product

b) balance of payment balance of trade

3. Qu'est-ce qu'un cartel ? Quels sont les trois genres de cartel ?

4. Comment calcule-t-on l'Indice des prix à la consommation?

5. Le chômage conjoncturel et le chômage structurel sont les deux sortes de chômage involontaire. Définissez-les. 
6. Traduisez le passage suivant: The average yield of the Toronto 300 index declined from 4,10\% on July 9 to 3,88\% on September 7 with the yield on the oil and gas index dropping to $1,24 \%$ from $1,37 \%$.

Il y aurait encore beaucoup à dire au sujet de la méthode par objectifs d'apprentissage préconisée ci-dessus. J'espère tout au moins que cet aperçu général aura permis d'en faire voir les nombreux avantages pédagogiques. Je peux dire, en terminant, que la réaction des étudiants à l'égard de cette méthode est très positive et très encourageante. Bien que la charge de travail exigée d'eux soit assez lourde, ils n'hésitent pas à fournir l'effort requis. Cela s'explique, je crois, par le fait qu'ils ont le sentiment de réaliser des progrès du double point de vue des connaissances acquises et de l'aptitude à traduire. Et cẻ double acquis se reflète dans les travaux pratiques dont la qualité augmente au fur et à mesure que le séminaire progresse. Et ça, c'est gratifiant pour le professeur!

\section{TABLEAU RÉCAPITULATIF}

Procédés d'apprentissage de

l'initiation à la traduction économique

Dans la salle de classe

- Exposés magistraux sur les notions

d'économie

- Vocabulaire photocopié des

mots clés de l'économie

- Exposés sur les difficultés

terminologiques

- Exercices de phraséologie

- Présentation des sources

documentaires

- Exposés sur les difficultés

grammaticales et stylistiques

- Discussion et commentaire des

traductions annotées

- Remise de corrigés (lus et

commentés)

m Journées de traduction*
En dehors de la salle de classe

- Lecture d'un ouvrage de

vulgarisation

- Aides audiovisuelles

- Renvoi à des études terminologiques publiées

- Recherches terminologiques

- Lectures parallèles

- Recherches documentaires

- Traduction individuelle de textes économiques

* Ces techniques peuvent être appliquées indifféremment dans ou en dehors de la salle de classe.

Notes et références

1. La Traduction : l'universitaire et le praticien. Actes du $23^{\mathrm{e}}$ congrès de l'APFUCC, publié sous la direction d'Arlette Thomas et de Jacques Flamand, "Cahiers de traductologie ", n 5 , Ottawa, Éditions de l'Université, 1984, p. 296.

2. Paru en 1987 sous le titre la Traduction au Canada/Translation in Canada, 1534-1984.

3. À deux reprises, au cours des cinq dernières années, j'ai vainement tenté d'organiser un tel colloque ; toutes sortes de raisons administratives et personnelles m'en ont empêché.

4. L'Analyse du discours comme méthode de traduction. Initiation à la traduction française de textes pragmatiques anglais, "Cahiers de traductologie", $n^{\circ} 2$, Ottawa, Éditions de l'Université, 1980, 282 p. Une $3^{e}$ éd. est en préparation.

5. Robert Galisson et Daniel Coste (1976) : Dictionnaire de didactique des langues, Paris, Hachette, p. 381. (Les italiques sont de moi.)

6. Ibid., p. 442. (Les italiques sont de moi.)

7. Denis Blondin, Service pédagogique, Université de Montréal, cité dans Info pédagogique, Université d'Ottawa, vol. $6, \mathrm{n}^{\circ} 2,1984$, p. 8. 
8. Cette question a été étudiée par Claude Simard dans une thèse inédite présentée à l'Université Laval pour l'obtention du grade de Maître ès arts (maîtrise en terminologie et traduction), Études des problèmes liés à la traduction économique, avril $1980,119 \mathrm{p}$.

9. Cahiers de l'Académie canadienne-française, $\mathrm{n}^{\circ} 15$ - Victor Barbeau, hommages et tributs, Montréal, Fides, 1978.

10. "Cooccurrence : Coexistence dans un même énoncé de deux ou plusieurs unités linguistiques distinctes, consécutives ou non. La cooccurrence est plus générale que la 'collocation', qui se limite à la coexistence d'unités consécutives. "R. Galisson et D. Coste, op. cit., p. 129.

11. L'unité textuelle est la portion de texte offrant un contexte suffisant pour permettre la recherche d'une équivalence de traduction d'une expression donnée ou d'un passage. Par contexte suffisant, il faut entendre la portion de texte de longueur variable fournissant l'information nécessaire pour traduire une unité lexicale, un énoncé, un paragraphe ou pour illustrer une difficulté quelconque de traduction. L'Analyse du discours comme méthode de traduction, pp. 149-150.

12. J. Delattre et $\mathrm{G}$. de Vernisy (1958) : le Vocabulaire baromètre dans le langage économique, Genève, Librairie de l'Université, p. 7.

13. Jean-Claude Gémar et Paul Horguelin (1977) : Bibliographie sélective du traducteur - Commerce et économie, Montréal, Linguatech, p. 2.

14. J.-P. Bénard et P. Horguelin (1977) : Pratique de la traduction. Version générale. Corrigés, Montréal, Linguatech, pp. 3-4.

15. Robert Larose (1984) : "Le rôle des annotations de texte dans l'enseignement de la traduction ", $M E T A$, $29: 2$, juin, p. 146 .

16. Pour plus de détails, voir l'article de Daniel Gouadec (1979) : "Journée de traduction, stage interne", META, 24:4, pp. 423-433.

17. L'Analyse du discours comme méthode de traduction, p. 236. 